\title{
Dependência espacial dos atributos físicos de três classes de solos cultivados com cana-de-açúcar sob colheita mecanizada
}

\author{
Laércio A. de Carvalho', Ismael Meurer ${ }^{2}$, Carlos A. da Silva Junior ${ }^{2}$, \\ Karina M. V. Cavalieri ${ }^{3} \&$ Cristiane F. B. Santos ${ }^{4}$
}

\begin{abstract}
RESU M 0
O bjetivou-se, neste trabalho, avaliar as propriedades físicas de três classes de solo cultivadas com cana-deaçúcar em sistema de colheita mecanizada na região de Mato Grosso do Sul. O local de estudo pertence à U sina Eldorado, Grupo ETH Bioenergia S/A, localizada no município de Rio Brilhante, MS. A amostragem em cada área experimental foi realizada a partir de uma malha, com $180 \mathrm{~m}$ de comprimento e 145,6 m de largura, totalizando 90 pontos em forma de grade, com 9 colunas e 10 linhas, onde foram coletadas amostras de estrutura indeformada nas camadas de 0-0,2 $\mathrm{m}$ e 0,2-0,4 $\mathrm{m}$ de profundidade nas entrelinhas da cultura. A pós coleta, as amostras foram processadas para determinação da densidade do solo (DS), porosidade total (PT) e umidade do solo $(\theta)$ para interpretação dos dados. A palhada deixada sobre superfície auxiliou na mantença de bom nível de matéria orgânica, corroborando na formação, agregação e estabilidade de poros, impedindo o aumento da densidade. A redução da porosidade total e consecutivo aumento na densidade do solo, principalmente na profundidade de 0,2-0,4 $\mathrm{m}$, indicam compactação nesta camada, justificada pelo intenso tráfego de veículos e ausência de revolvimento do solo.
\end{abstract}

Palavras-chave: Saccharum officinarum, densidade do solo, geoestatística, estabilidade de agregados

\section{Physical properties of three classes of soils cultivated with sugarcane in mechanized harvesting}

ABSTRACT

The aim of this study was to evaluate the physical properties of three soils cultivated with sugarcane mechanized harvest system in the region of $M$ ato Grosso do Sul The study site belongs to the Plant Eldorado, ETH Bioenergy Group S/A, located in Rio Brilhante, MS. The sampling in each experimental area was made from a grid with $180 \mathrm{~m}$ long and $145.6 \mathrm{~m}$ wide, totaling 90 points in a grid form, with nine columns and 10 rows, where samples were collected in undisturbed layers of 0-0.2 $\mathrm{m}$ and $0.2-0.4 \mathrm{~m}$ deep in the rows. After collection, samples were processed for determination of soil bulk density (BD), total porosity (TP) and soil moisture $(\theta)$ for data interpretation. Crop residues left on surface helped to maintain good level of organic matter, supporting the formation, aggregation and stability of pores, preventing the increase in density. The reduction in total porosity and consecutive increase in density, especially in the 0.2-0.4 m, indicating compaction of this layer, justified by the intense traffic of vehicles and lack of soil disturbance.

Key words: Saccharum officinarum, bulk density, geostatistics, aggregate stability

UEMS, Dourados, MS. CP 351, CEP 79804-970. Fone: (67) 3902-2660. E-mail: Icarvalh@uems.br

${ }^{2}$ Graduando em Agronomia/U EMS - Rodovia Aquidauana/CERA - km 12, CP 25, CEP 79200-000, Aquidauana, MS. Fone: (67) $3904-2902$. E-mails: carlos-junior89@hotmail.com; ismael_meurer@yahoo.com.br

${ }^{3}$ D epartamento de Solos e Eng. Agrícola, D SEA/U EM S, Rua dos Funcionários, 1540, CEP 80035-050, Curitiba, PR. Fone: (41) 3350-5655. E-mail: karina_cavalieri@yahoo.com.br

${ }^{4}$ Pós-G raduanda em Recursos N aturais/U EM S, CP 351, CEP 79804-970, D ourados, MS. Fone: (67) 3902-2660. E-mail: cristiane.ferrari@yahoo.com.br 


\section{INTRODUÇÃO}

A busca por alternativas que proporcionem a sustentabilidade do ambiente agrícola e minimização dos custos de produção vem mudando o comportamento das unidades produtoras de cana-de-açúcar (Souza et al., 2005). O manejo de colheita com queima, comumente utilizado para eliminar o grande volume de matéria seca no dossel da planta está sendo substituído pelo manejo de cana crua, caracterizado pelo uso de máquinas para realizar a colheita, o que evita a contaminação do ambiente e de centros urbanos e ainda traz maior eficiência e velocidade na operação (Spadoto, 2008).

Observa-se, porém, uma intensa degradação da qualidade física do solo considerando-se que o tráfego desses equipamentos de forma repetitiva, induz a mudanças no comportamento das partículas dos agregados do solo, ocasionando a deterioração de sua estrutura provocando, assim, o processo de compactação do solo (Iaia et al., 2006), fenômeno que se agrava quando o solo é trabalhado com um conteúdo de água elevado, inadequado para as atividades agrícolas (Silva \& Cabeda, 2006).

As modificações que ocorrem na estrutura do solo, promovidas pela compactação são evidenciadas por alterações nos valores de vários atributos, como densidade do solo, resistência mecânica à penetração, porosidade total, porosidade de aeração, armazenagem e disponibilidade de água às plantas, dinâmica de água na superfície e no seu perfil, assim como na consistência e na máxima compactabilidade do solo (Klein et al., 1998).

Figueiredo et al. (2000), definem que a compactação é uma consequência direta do manejo inadequado e a umidade é o fator que controla a quantidade de deformação que poderá ocorrer no solo.

Para Marsili et al. (1998), o processo de compactação pode ser atribuído tanto à diminuição do espaço poroso entre os agregados, ocorrendo um rearranjamento na matriz do solo, como à ruptura e destruição dos agregados, havendo rearranjamento e orientação das partículas, o que resulta numa massa coesa na matriz do solo.

As atividades agrícolas devem ser realizadas com o solo no estado de friabilidade, que corresponde à faixa de umidade entre os limites de contração e plasticidade. Neste estado, o solo está úmido, demonstrando as condições ideais de preparo e/ou colheita mecanizada sendo, portanto, a faixa de friabilidade definida como a faixa ideal para as atividades (Silva et al., 2006).

Assim, com a caracterização da camada compactada e do comportamento do solo em relação às suas propriedades físicas, como densidade, porosidade, umidade e capacidade de retenção e infiltração da água, pode-se adotar um sistema de manejo ideal para aquela determinada classe de solo, enfatizando a admissão de um status conservacionista (Vieira $\&$ Klein, 2007).

Objetivou-se, neste trabalho, avaliar as propriedades físicas de três classes de solo cultivadas com cana-de-açúcar em sistema de colheita mecanizada, no estado de Mato Grosso do Sul.

\section{MATERIAL E MÉTODOS}

O local de estudo pertence à Usina Eldorado - Grupo ETH Bioenergia S/A, localizada no município de Rio Brilhante, MS, e inserida na Bacia do Rio Paraná, subbacia do Rio Ivinhema, cujas coordenadas estão situadas a $21^{\circ} 50^{\prime}, 52^{\prime \prime}$ de Latitude sul e 53 57' 49" de Longitude oeste, com altitude média de $650 \mathrm{~m}$.

O clima da região é caracterizado tipo Aw, tropical de savana com período chuvoso concentrado no verão e estação seca no inverno, de acordo com a classificação de Köppen, com precipitação média de $1440 \mathrm{~mm}$ ao ano.

O estudo foi conduzido em três classes de solo (Tabela 1), classificados conforme metodologia da EMBRAPA (2006), sendo o conteúdo de matéria orgânica determinado de acordo com van Raij et al. (2001).

Tabela 1. Composição granulométrica nas duas camadas de solos estudados

\begin{tabular}{|c|c|c|c|c|c|c|}
\hline \multirow{2}{*}{ Área } & Argila & Silte & Areia & $\mathrm{CO}$ & \multirow{2}{*}{$\begin{array}{c}\text { DP } \\
\mathrm{g} \mathrm{cm}^{-3}\end{array}$} & \multirow{2}{*}{ Classe textural } \\
\hline & \multicolumn{4}{|c|}{$\mathrm{g} \mathrm{kg}^{-1}$} & & \\
\hline \multicolumn{7}{|c|}{ Camada de 0-0,20 m } \\
\hline 1 & 292 & 69 & 639 & 13,01 & 2,74 & Franco argilo arenosa \\
\hline 2 & 501 & 79 & 420 & 15,73 & 2,88 & Argilosa \\
\hline 3 & 112 & 47 & 841 & 9,99 & 2,72 & Areia franca \\
\hline \multicolumn{7}{|c|}{ Camada de $0,20-0,40 \mathrm{~m}$} \\
\hline 1 & 365 & 59 & 576 & 6,35 & 2,77 & Argilo arenosa \\
\hline 2 & 589 & 92 & 320 & 8,29 & 2,91 & Argilosa \\
\hline 3 & 201 & 39 & 760 & 4,10 & 2,74 & Franco argilo arenosa \\
\hline
\end{tabular}

O preparo das áreas se iniciou em 2005, com gradagem aradora e aração para incorporação de calcário e gesso, havendo ainda uma gradagem niveladora e sulcação para o plantio da cana.

Nos quatro ciclos de cultivo da cana-de-açúcar que antecederam a amostragem, em fevereiro de 2010, foi realizada a colheita da cana crua, de forma mecanizada, utilizando-se colhedora com peso aproximado de 16 ton junto a um transbordo acoplado a um trator com carga máxima total de 20 ton.

A amostragem em cada uma das três áreas, foi realizada a partir de uma malha experimental com $180 \mathrm{~m}$ de comprimento e 145,6 m de largura, totalizando 90 pontos distanciados $20 \mathrm{~m}$ entre si no eixo x e 18,2 m no eixo y, demarcados com fita métrica, com 9 colunas e 10 linhas, na qual foram coletadas amostras de estrutura indeformada na camada de 0-0,2 m e 0,2-0,4 m de profundidade nas entrelinhas da cultura.

Para a coleta das amostras foram utilizados anéis metálicos com dimensão de 0,051 m de diâmetro e 0,05 m de altura, os quais foram inseridos no amostrador do tipo Uhland para a retirada das amostras do solo. Assim que estas eram coletadas, eram envoltas em papel alumínio, acondicionadas em sacos plásticos e mantidas sob temperatura de aproximadamente 5 ${ }^{\circ} \mathrm{C}$ até serem processadas.

A amostra úmida retirada do campo foi limpa, retirando-se o excesso de solo, pesada e, em seguida, colocada em estufa a uma temperatura de $105^{\circ} \mathrm{C}$ até atingir peso constante. 
Permitiu-se, então, a determinação da densidade de partículas pelo método do balão volumétrico, descrito pela EMBRAPA (1997), densidade do solo, umidade e porosidade total, através das Eqs. 1, 2 e 3, respectivamente.

$$
\mathrm{DS}=\frac{\mathrm{Mss}}{\mathrm{Vt}}
$$

em que:

DS - densidade do solo, $\mathrm{g} \mathrm{cm}^{-3}$

Mss - massa do solo seco, $\mathrm{g}$

$\mathrm{Vt}$ - volume total, $\mathrm{cm}^{3}$

$$
\theta=\left(\frac{\mathrm{Msu}-\mathrm{Mss}}{\mathrm{Vt}}\right) \cdot 100
$$

onde:

$\theta \quad$ - umidade do solo, $\mathrm{m}^{3} \mathrm{~m}^{-3}$

Msu - massa do solo úmido, $\mathrm{Mg}$

Mss - massa do solo seco, $\mathrm{Mg}$

$\mathrm{Vt}$ - volume total, $\mathrm{m}^{3}$

$$
\mathrm{PT}=\left(1-\frac{\mathrm{DS}}{\mathrm{DP}}\right) \cdot 100
$$

sendo:

$$
\begin{aligned}
& \text { PT - porosidade total, } \% \\
& \text { DP - densidade de partículas, } \mathrm{g} \mathrm{cm}^{-3} \\
& \text { DS - densidade do solo, } \mathrm{g} \mathrm{cm}^{-3}
\end{aligned}
$$

A análise estatística descritiva foi feita com auxilio do programa exploratório Statistica 4.3 (Statsoft, 2005), a fim de observar o comportamento geral dos dados e identificar prováveis discrepâncias.

Aplicou-se, ainda, o teste paramétrico de normalidade de Shapiro \& Wilk (1965) e não-paramétrico de normalidade de Kolmogorov Smirnov (KS), obtidos no mesmo programa.

Os atributos que apresentaram valores discrepantes foram descartados e a análise foi refeita. Após a análise descritiva, semivariogramas foram construídos para observar o grau de dependência espacial dos atributos em estudo, utilizando-se o aplicativo GS+ (versão 7.0) (Gamma Design Software, 2004).

\section{RESULTADOS E DISCUSSÃO}

Na Tabela 2 são apresentados os valores obtidos das medidas estatísticas descritivas para a Área 1, 2 e 3, nas quais se encontram as variáveis densidade do solo, umidade e porosidade total, nas duas camadas estudadas.

Observa-se, na Tabela 2, que os valores de média e mediana para os atributos DS, $\theta$ e PT foram semelhantes, indicando uma distribuição normal dos dados, o que também pode ser confirmado pelos valores do teste de Shapiro e Wilk ( $p<0,05)$ e assimetria, próximos de zero, apesar da ocorrência de algumas distribuições assimétricas.
Estes resultados concordam com os obtidos por Corá \& Beraldo (2006), Gontijo et al. (2008) e Cruz et al. (2010), indicando que os dados estão adequados para o uso da geoestatística.

O teste de Kolmogorov Smirnov não indicou normalidade nos dados, visto que estão fora do nível de $5 \%$ de probabilidade, discordando de Souza et al. (2010) que encontraram normalidade para esses parâmetros.

Segundo Ortiz (2003), os coeficientes de curtose são utilizados para avaliar se os dados seguem uma distribuição normal. Os valores, obtidos por meio do software Statistica 4.3 (Statsoft, 2005) devem ser nulos, sendo aceitos valores entre $+2 \mathrm{e}-2$.

Analisando a área 1 da Tabela 2 observa-se que, para todas as variáveis e camadas, os valores de curtose estão fora dos limites aceitos por Ortiz (2003).

Este fato não é encontrado nas áreas 2 e 3 da Tabelas 2 que, apesar de apresentarem amostras de umidade na segunda camada fora do aceito, seguem um padrão normal, concordando com Corá \& Beraldo (2006), que, analisando a densidade e porosidade total em Latossolo Vermelho cultivado com canade-açúcar, obtiveram resultados dentro dos limites aceitos por Ortiz(2003).

De acordo com Berner et al. (2006), a variabilidade dos dados medidos é descrita pela estimativa da variância e pelo coeficiente de variação (CV). A variabilidade dos atributos do solo (Tabela 2) medida pelo coeficiente de variação pode ser considerada a primeira indicação da existência de heterogeneidade nos dados.

Neste caso e de acordo com a classificação de Warrick \& Nielsen (1980), os resultados demonstraram alto CV (>24\%) para a variável umidade na camada de 0,2-0,4 m na Tabela 2, área 1, e ambas as camadas da mesma variável na área 3, discordando de Souza et al. (2006a), que obtiveram CV baixo para a variável umidade em cana-de-açúcar.

Na Tabela 2, área 1, se obteve ainda, CV médio (12-24\%), para a variável umidade na camada $0-0,2 \mathrm{~m}$, na variável porosidade total na camada $0,2-0,4 \mathrm{~m}$ e nas duas camadas da variável umidade na área 2 (Tabela 2). Timm et al. (2004) e Siqueira et al. (2008) confirmam coeficientes de variação semelhantes para umidade.

As variáveis densidade e porosidade total apresentaram CV de caráter baixo $(<12 \%)$, em todas as camadas e Áreas. Souza et al. (2006b) e Ramirez-López et al. (2008) também encontraram CV baixo para estas variáveis.

De forma geral, a dispersão das medidas em torno da média variou de 3,95\% (variável densidade, camada de 0,2-0,4 m, Tabela 4) a 33,33\% (variável umidade, camada de 0,2-0,4 m, Tabela 4), dados também obtidos por Sanchez et al. (2009), Corá \& Beraldo (2006) e Mello et al. (2006).

Observa-se que a densidade do solo sofre um pequeno acréscimo com a profundidade, em todas as áreas, variando de 1,38 a $1,66 \mathrm{~g} \mathrm{~cm}^{-3}$ para a camada de $0-0,2 \mathrm{~m} \mathrm{e} 1,42$ a $1,77 \mathrm{~g} \mathrm{~cm}^{-3}$ para camada $0,2-0,4 \mathrm{~m}$.

O aumento da densidade sugere uma possível compactação subsuperficial devido ao tráfego de veículos pesados na área, por ocasião da colheita da cana-de-açúcar e ausência de mobilização mecânica do solo, fato também encontrado por Paulino et al. (2004), Klein \& Libardi (2002) e Tormena et al. 
Tabela 2. Resumo estatístico para todas as variáveis nas duas profundidades das Áreas 1,2 e 3

\begin{tabular}{|c|c|c|c|c|c|c|}
\hline \multirow{2}{*}{$\begin{array}{c}\text { Variáveis } \\
\text { Medidas/Profundidade }\end{array}$} & \multicolumn{2}{|c|}{$\operatorname{DS}\left(\mathrm{g} \mathrm{cm}^{-3}\right)$} & \multicolumn{2}{|c|}{$\theta\left(\mathrm{m}^{3} \mathrm{~m}^{-3}\right)$} & \multicolumn{2}{|c|}{ PT (\%) } \\
\hline & $0-0,2 \mathrm{~m}$ & $0,2-0,4 \mathrm{~m}$ & $0-0,2 \mathrm{~m}$ & $0,2-0,4 \mathrm{~m}$ & $0-0,2 \mathrm{~m}$ & $0,2-0,4 \mathrm{~m}$ \\
\hline & \multicolumn{6}{|c|}{ Área 1} \\
\hline Média & 1,63 & 1,71 & 0,35 & 0,36 & 40,52 & 38,28 \\
\hline Mediana & 1,63 & 1,74 & 0,34 & 0,35 & 40,45 & 37,16 \\
\hline Mínimo & 1,12 & 1,12 & 0,26 & 0,26 & 33,25 & 32,48 \\
\hline Máximo & 1,83 & 1,87 & 0,84 & 0,93 & 59,15 & 59,66 \\
\hline Quartil inferior & 1,59 & 1,68 & 0,32 & 0,32 & 38,24 & 35,67 \\
\hline Quartil superior & 1,69 & 1,78 & 0,36 & 0,36 & 41,95 & 39,15 \\
\hline Variância & 0,01 & 0,02 & 0,00 & 0,01 & 14,59 & 22,51 \\
\hline Desvio padrão & 0,10 & 0,13 & 0,07 & 0,09 & 3,82 & 4,74 \\
\hline Curtose & 5,53 & 10,18 & 35,54 & 29,71 & 5,53 & 10,18 \\
\hline Coef. Var. (\%) & 6,13 & 7,60 & 20,00 & 25,00 & 9,43 & 12,38 \\
\hline Assimetria & $-1,41$ & $-2,91$ & 5,04 & 5,05 & 1,41 & 2,91 \\
\hline Shapiro \& Wilk & 0,00 & 0,00 & 0,00 & 0,00 & 0,00 & 0,00 \\
\hline \multirow[t]{2}{*}{ Kolmogorov Smirnov } & 0,12 & 0,22 & 0,32 & 0,34 & 0,11 & 0,22 \\
\hline & \multicolumn{6}{|c|}{ Área 2} \\
\hline Média & 1,38 & 1,42 & 0,36 & 0,38 & 52,02 & 51,12 \\
\hline Mediana & 1,38 & 1,47 & 0,35 & 0,39 & 52,17 & 49,61 \\
\hline Mínimo & 1,10 & 1,04 & 0,28 & 0,11 & 43,98 & 43,19 \\
\hline Máximo & 1,61 & 1,65 & 0,52 & 0,97 & 61,79 & 64,43 \\
\hline Quartil inferior & 1,29 & 1,33 & 0,33 & 0,34 & 48,42 & 46,68 \\
\hline Quartil superior & 1,48 & 1,54 & 0,38 & 0,41 & 54,96 & 54,40 \\
\hline Variância & 0,01 & 0,02 & 0,00 & 0,01 & 17,22 & 25,56 \\
\hline Desvio padrão & 0,12 & 0,15 & 0,04 & 0,09 & 4,15 & 5,07 \\
\hline Curtose & $-0,90$ & $-0,42$ & 2,55 & 22,93 & $-0,90$ & $-0,42$ \\
\hline Coef. Var. (\%) & 8,70 & 10,56 & 11,11 & 23,68 & 7,98 & 9,92 \\
\hline Assimetria & $-0,01$ & $-0,61$ & 1,08 & 2,99 & 0,01 & 0,61 \\
\hline Shapiro \& Wilk & 0,03 & 0,00 & 0,00 & 0,00 & 0,05 & 0,00 \\
\hline \multirow[t]{2}{*}{ Kolmogorov Smirnov } & 0,07 & 0,14 & 0,12 & 0,21 & 0,06 & 0,14 \\
\hline & \multicolumn{6}{|c|}{ Área 3} \\
\hline Média & 1,66 & 1,77 & 0,16 & 0,18 & 38,87 & 35,37 \\
\hline Mediana & 1,66 & 1,78 & 0,16 & 0,17 & 39,00 & 35,20 \\
\hline Mínimo & 1,43 & 1,51 & 0,06 & 0,05 & 31,32 & 30,54 \\
\hline Máximo & 1,87 & 1,9 & 0,31 & 0,51 & 47,56 & 45,01 \\
\hline Quartil inferior & 1,60 & 1,72 & 0,13 & 0,14 & 36,52 & 33,62 \\
\hline Quartil superior & 1,72 & 1,82 & 0,20 & 0,20 & 41,02 & 36,98 \\
\hline Variância & 0,01 & 0,01 & 0,00 & 0,00 & 12,24 & 6,50 \\
\hline Desvio padrão & 0,10 & 0,07 & 0,05 & 0,06 & 3,50 & 2,55 \\
\hline Curtose & 0,24 & 2,11 & 0,23 & 9,87 & 0,02 & 2,11 \\
\hline Coef. Var. (\%) & 6,02 & 3,95 & 31,25 & 33,33 & 9,00 & 7,21 \\
\hline Assimetria & $-0,68$ & $-0,90$ & 0,60 & 2,13 & 0,07 & 0,90 \\
\hline Shapiro \& Wilk & 0,42 & 0,01 & 0,18 & 0,00 & 0,45 & 0,01 \\
\hline Kolmogorov Smirnov & 0,06 & 0,08 & 0,09 & 0,13 & 0,05 & 0,07 \\
\hline
\end{tabular}

DS - Densidade do solo; $\theta$ - umidade; PT - porosidade total

(2002). Silva et al. (2000) explicam que o aumento da largura dos pneus não acompanhou a mecanização das lavouras, motivo que aumenta o risco de compactação.

Michelon (2005) define alguns níveis de densidade do solo estabelecidos como críticos para indicar a ocorrência de compactação, levando em consideração a faixa do teor de argila. Neste caso, para teores de 0-200, 200-300, 300-400, 400-500 e 500-600 $\mathrm{g} \mathrm{kg}^{-1}$ a densidade crítica deverá ser de 1,60, 1,55, 1,50, 1,45 e $1,40 \mathrm{~g} \mathrm{~cm}^{-3}$, respectivamente.

Estudando a granulometria dos solos presente na Tabela 1 e relacionando-a com os resultados da variável densidade nas Tabelas 2, 3 e 4, observa-se que apenas a primeira camada da Área 2 apresenta densidade dentro dos limites críticos propostos por Michelon (2005), permanecendo os demais sempre acima do permitido.

Fernandes et al. (1983) explicam que em Latossolo Vermelho valores de densidade superiores a $1,45 \mathrm{~g} \mathrm{~cm}^{-3}$ prejudicam a absorção de nutrientes pela planta. Segundo Reichardt \& Timm (2004) e Libardi (2005), solos arenosos apresentam densidades superiores a dos solos argilosos, enquanto os solos siltosos apresentam comportamento intermediário. A densidade dos solos arenosos pode variar de 1,3 a $1,8 \mathrm{~g} \mathrm{~cm}^{-3}$, enquanto em solos argilosos esta variação pode ir de 0,9 a $1,6 \mathrm{~g} \mathrm{~cm}^{-3}$.

Souza et al. (2005) encontraram maior interação entre as frações orgânica e mineral do solo, assim como a melhor estabilidade de agregados em Latossolo sob manejo de cana crua devido, principalmente, à adição de cerca de $12 \mathrm{t} \mathrm{ha}^{-1} \mathrm{de}$ matéria orgânica na forma de palhada. Costa et al. (2004) observaram que o maior teor de matéria orgânica determina maior estabilidade dos agregados e, consequentemente, maior proteção física.

A presença de maiores índices de umidade e matéria orgânica nos solos estudados pode ter favorecido a floculação das partículas em agregados estáveis, porém, o tempo de cultivo 
Tabela 3. Grau de dependência espacial para os semivariogramas experimentais das variáveis densidade (DS), umidade ( $\theta$ ) e porosidade total (PT), na profundidade de 0-0,2 m e 0,2-0,4 $\mathrm{m}$, nas três áreas estudadas

\begin{tabular}{|c|c|c|c|c|c|c|}
\hline \multirow{2}{*}{$\begin{array}{l}\text { Variáveis } \\
\text { Profundidade }\end{array}$} & \multicolumn{2}{|c|}{ DS } & \multicolumn{2}{|c|}{$\boldsymbol{\theta}$} & \multicolumn{2}{|c|}{ PT } \\
\hline & $0-0,2 \mathrm{~m}$ & $0,2-0,4 \mathrm{~m}$ & $0-0,2 \mathrm{~m}$ & $0,2-0,4 \mathrm{~m}$ & $0-0,2 \mathrm{~m}$ & $0,2-0,4 \mathrm{~m}$ \\
\hline $\begin{array}{c}\text { Parâmetro } \\
\left(\mathrm{Co} /\left(\mathrm{C}_{0}+\mathrm{C}_{1}\right)\right) \times 100\end{array}$ & 0,1040 & 0,0762 & 0,2545 & 0,1477 & 0,0782 & 0,0584 \\
\hline$\left(\mathrm{CO} /\left(\mathrm{CO}_{0}+\mathrm{C}_{1}\right)\right) \times 100$ & 0,0632 & - & 3,3149 & 4,2683 & 0,0523 & - \\
\hline$\left(\mathrm{Co} /\left(\mathrm{C}_{0}+\mathrm{C}_{1}\right)\right) \times 100$ & 6,5611 & 0,2304 & - & - & 6,9082 & 0,1733 \\
\hline
\end{tabular}

PT - porosidade total; DS - densidade do solo; $\theta$ - umidade; (-) - efeito pepita puro

da cana-de-açúcar propiciou maior aumento na densidade do solo, como encontrado por Centurion et al. (2007) em Latossolo Vermelho. Dados semelhantes foram estudados por Camilotti et al. (2005), na mesma classe de solo, ao observarem compactação em subsuperfície após quatro anos de sucessão de ciclos da cana-de-açúcar.

Reichert et al. (2007) definiram, através de análise do intervalo hídrico ótimo, que a densidade crítica para solos Argilosos varia de $1,30 \mathrm{a} 1,40 \mathrm{~g} \mathrm{~cm}^{-3}$. Analisando a densidade no Argissolo Vermelho (Tabela 4), observa-se um ligeiro aumento neste atributo, definindo grandes chances deste solo estar sofrendo compactação, o que também é observado pelo comportamento crítico do volume de água em relação aos demais.

Braida et al. (2010) explicam que em Argissolos o efeito da matéria orgânica sobre a resistência dos pontos de contato entre as partículas minerais não é tão significativo quanto em solos argilosos, uma vez que as partículas de areia apresentam uma reatividade muito menor que a das partículas de argila, impedindo o desenvolvimento de ligações fortes entre elas e a matéria orgânica; logo, frente a uma situação de compressibilidade um Argissolo terá maior facilidade em compactar frente a um Latossolo.

A porosidade total foi de 35,37\% em Argissolo (Área 3), $38,28 \%$ em Latossolo textura média (Área 1) e 51,12\% para Latossolo de textura argilosa (Área 2). Cruz et al., (2004), Camilotti et al. (2005), Paulino et al. (2004), Centurion et al. (2007) e Souza et al. (2004), obtiveram resultados semelhantes nesses solos.

Para Kiehl (1979) um solo ideal para a produção agrícola deve apresentar $0,50 \mathrm{~m}^{3} \mathrm{~m}^{-3}$ de porosidade total, sendo $1 / 3$ macroporos e 2/3 microporos. Ainda de acordo com o autor, a maioria das plantas desenvolve satisfatoriamente seu sistema radicular quando o volume de macroporos está acima de 0,10 $\mathrm{m}^{3} \mathrm{~m}^{-3}$. Relacionando as afirmações, compreende-se que um solo deve, então, apresentar uma porosidade total mínima de $30 \%$ para o crescimento radicular.

Neste estudo, apenas o Latossolo de textura argilosa apresentou condições ideais de porosidade total $(51,12 \%)$, sendo que o Argissolo e o Latossolo textura média permanecem em níveis considerados mínimos (35,37-38,28\%, respectivamente).

Silva \& Ribeiro (1997), estudando o efeito de vários anos de cultivo convencional de cana-de-açúcar verificaram que, nos solos com maior tempo de cultivo, a porosidade diminuía, com maior evidência para a macroporosidade, visto que esta representa os espaços entre os agregados mais afetados pelo manejo.
Segundo Carlesso \& Zimmermann (2000), em solos de textura média e argilosa, onde é comum a ocorrência de agregados porosos e elevado teor de matéria orgânica, os espaços porosos por unidade de volume são elevados; já em solos de textura arenosa a porosidade total é usualmente reduzida.

Observa-se, na Tabela 2, que a densidade tem relação inversamente proporcional à porosidade, uma vez que, quanto mais denso é o solo menor é sua porosidade; logo, se este apresentar densidade regular ou baixa, sua porosidade será maior. Pacheco (2010), Stone \& Silveira (2001) e Cruz et al. (2010), confirmam esta relação.

Uma vez que a porosidade total é formada pelo conjunto de macro e microporos, quaisquer alterações em suas estruturas afetam a porosidade, como um todo.

Tormena et al. (1998) confirmam que os macroporos são a classe de poros menos estáveis e sofrem colapso quando submetidos aos estresses aplicados pelos sistemas de preparo e tráfego, tornando-se facilmente instáveis, mediante compressibilidade, afetando a porosidade total.

Segundo Cruz et al. (2003) a estabilidade da estrutura depende da matéria orgânica, verificando-se menor risco de degradação estrutural e de compactação em solos com maiores teores de carbono orgânico (CO). Verificando a Tabela 1, constata-se que o Latossolo de textura argilosa apresenta maiores índices de $\mathrm{C}$ orgânico, podendo ser este o responsável por sua elevada porosidade.

Na Figura 1 se representam os semivariogramas experimentais e modelados para as variáveis densidade, umidade e porosidade total nas três classes de solos e duas camadas estudadas.

Os resultados da análise geoestatística demonstraram que as variáveis estudadas apresentaram dependência espacial nas três classes de solos e nas duas profundidades, demonstrando, porém efeito pepita puro (distribuição aleatória) nas variáveis $\theta$ (Figura 1H e 1Q), DS (Figura 1M) e PT (Figura 1O), indicando que para essas variáveis a distância de amostragem não foi capaz de exibir toda a variância dos dados e, provavelmente existe tendência nos dados em seguir determinada direção.

Em sua maioria os resultados dos dados da variável densidade do solo tiveram os semivariogramas ajustados ao modelo gaussiano (Figura 1A, 1D e 1J) obtendo-se ainda, modelo exponencial (Figura 1G), esférico (Figura 1P) e linear (Figura 1M).

Para a variável umidade os dados resultaram em maior ajuste no modelo exponencial (Figura 1B, 1H, 1Ke 1Q), tendo presença dos modelos gaussiano (Figura 1E) e esférico (Figura 1N). 

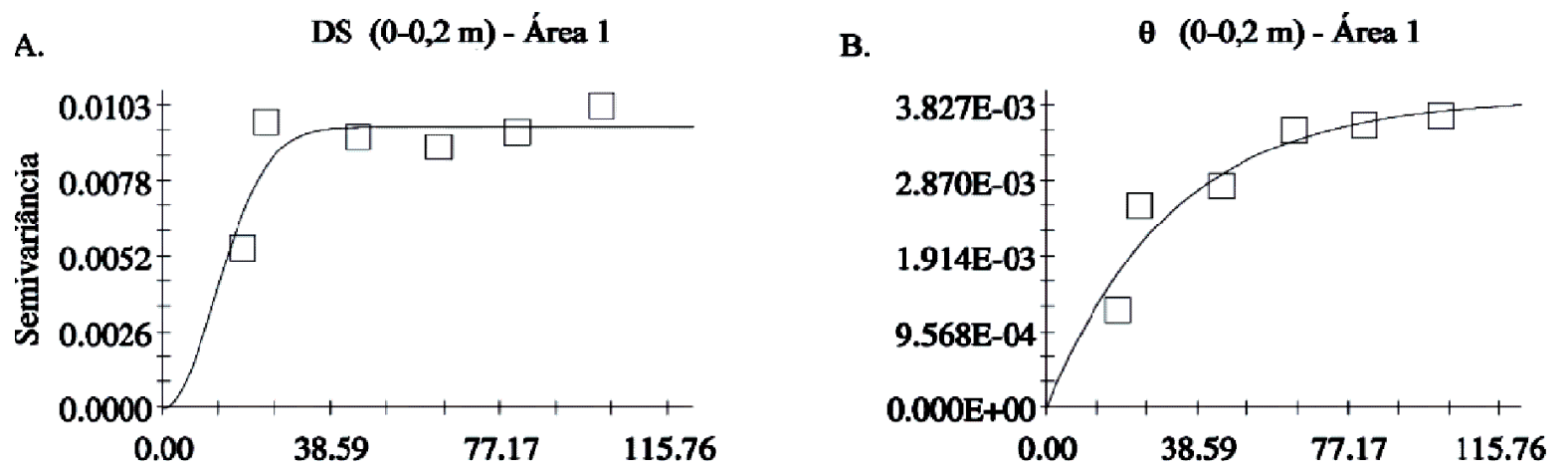

Gaussiano $\mathrm{Co}=0.00001 ; \mathrm{Co}+\mathrm{C}=0.00962 ; \mathrm{Ao}=16.8 ;$

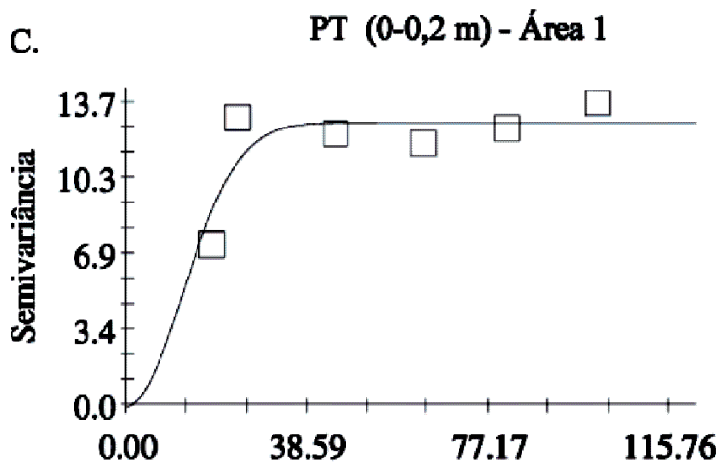

Exponencial $\mathrm{Co}=0.00001 ; \mathrm{Co}+\mathrm{C}=0.00393 ; \mathrm{Ao}=31.8$;

D. DS (0-0,2 m) - Área 2

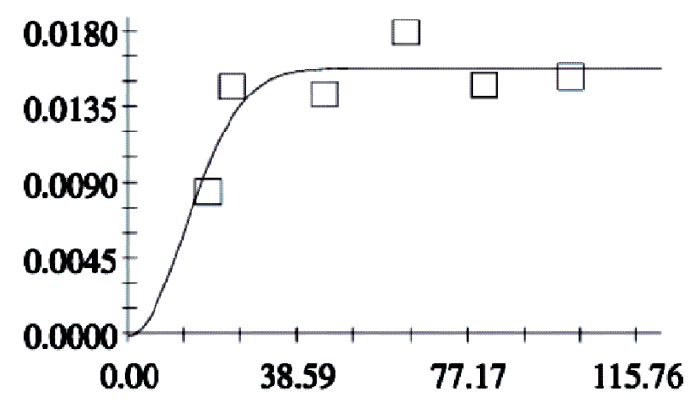

Gaussiano $\mathrm{Co}=0.01000 ; \mathrm{Co}+\mathrm{C}=12.79000 ; \mathrm{Ao}=16.8 ; \quad$ Gaussiano $\mathrm{Co}=0.00001 ; \mathrm{Co}+\mathrm{C}=0.01582 ; \mathrm{Ao}=18.1$;

E.

$\theta(0-0,2 \mathrm{~m})$ - Área 2

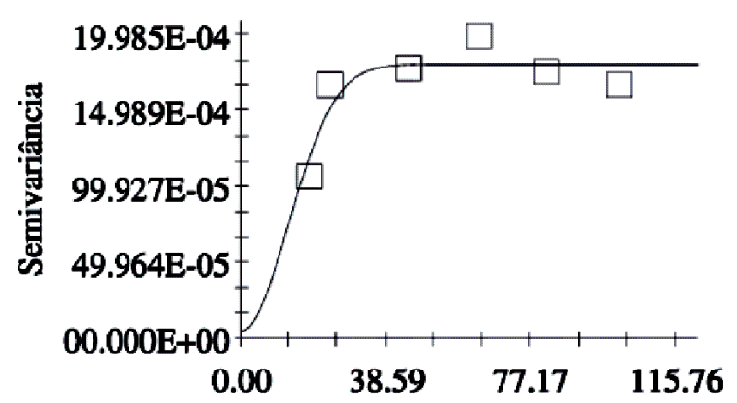

F.

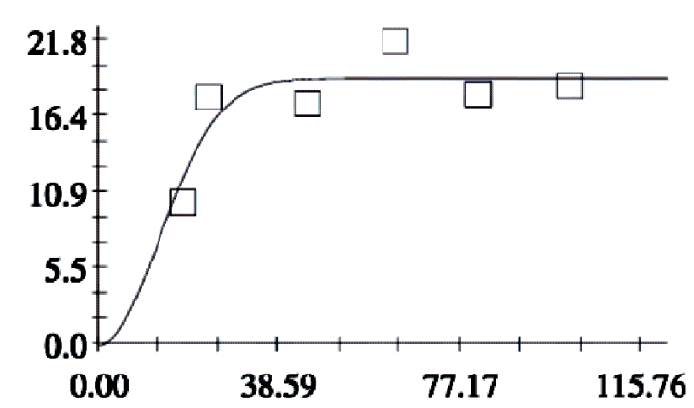

Gaussiano $\mathrm{Co}=0.00006 ; \mathrm{Co}+\mathrm{C}=0.00181 ; \mathrm{Ao}=17.7$;

G. DS (0-0,2 m) - Área 3

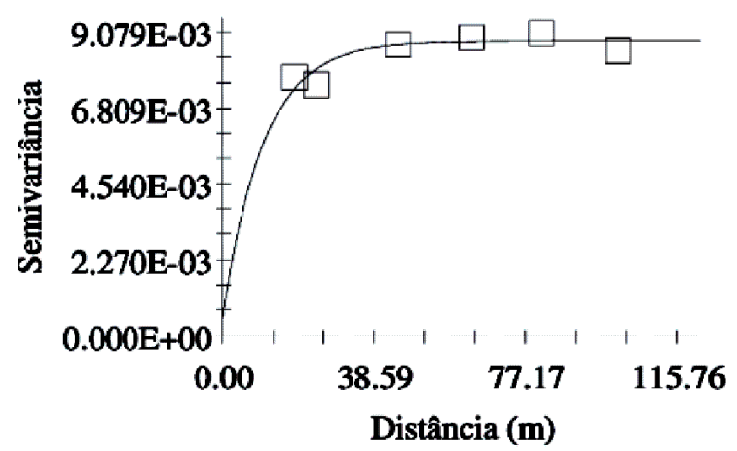

Gaussiano $\mathrm{Co}=0.01000 ; \mathrm{Co}+\mathrm{C}=19.12000 ; \mathrm{Ao}=18.2$;

H. 0 (0-0,2 m) - Area 3

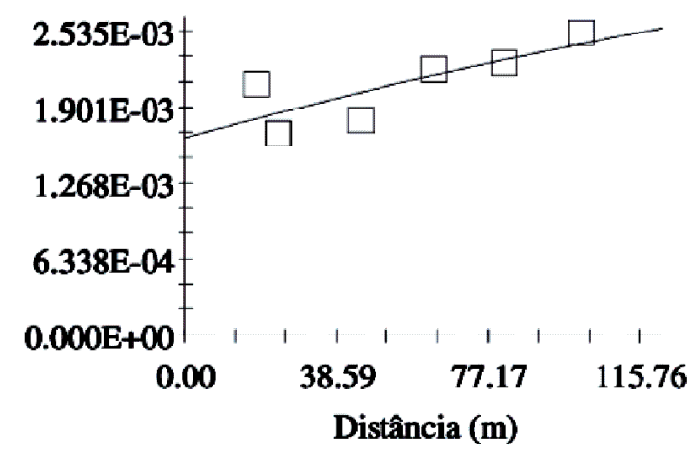

Exponencial $\mathrm{Co}=0.00166 ; \mathrm{Co}+\mathrm{C}=0.00438 ; \mathrm{Ao}=297.0$;

Continua na próxima página 
Continuação da Figura 1

I.

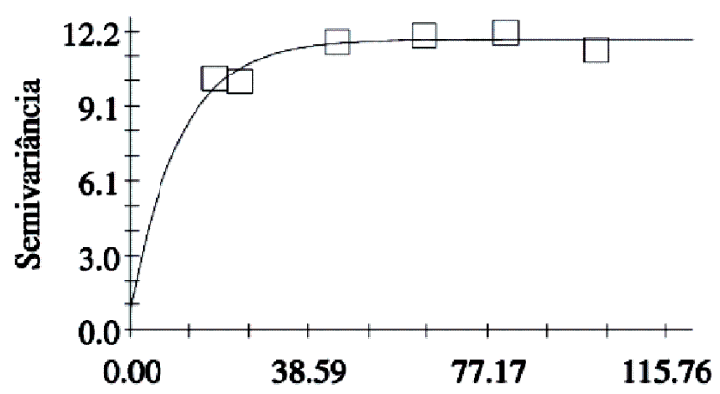

J.

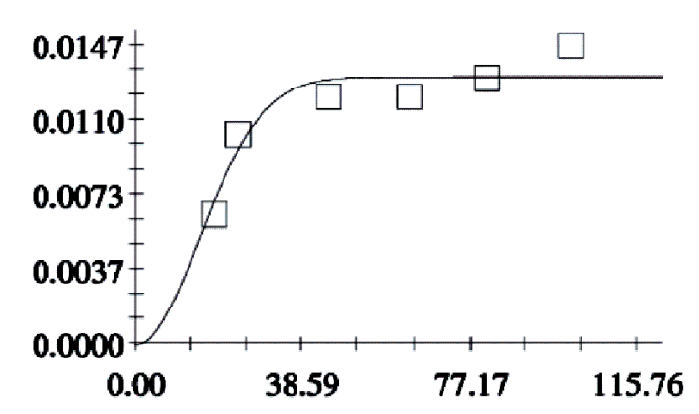

Exponencial $\mathrm{Co}=0.82000 ; \mathrm{Co}+\mathrm{C}=11.87000 ; \mathrm{Ao}=10.5 ; \quad$ Gaussiano $\mathrm{Co}=0.00001 ; \mathrm{Co}+\mathrm{C}=0.01312 ; \mathrm{Ao}=20.7$;

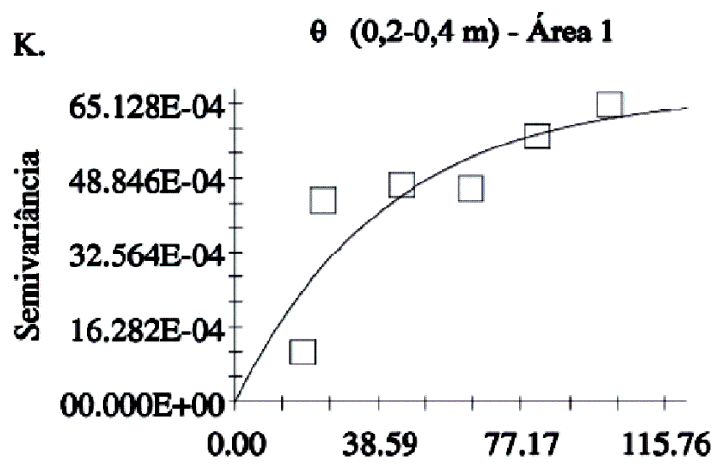

L.

PT $(0,2-0,4 \mathrm{~m})$ - Área 1

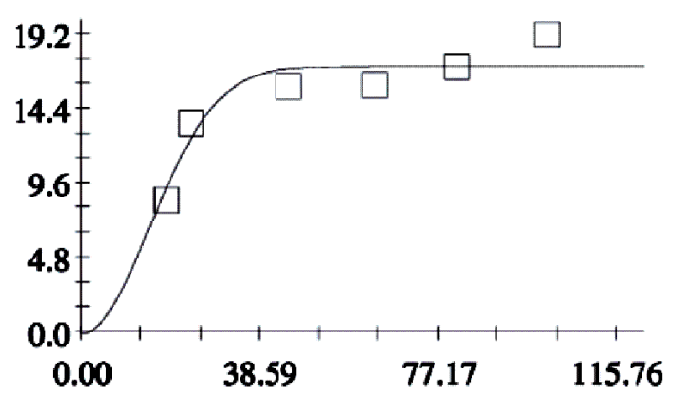

Exponencial $\mathrm{Co}=0.00001 ; \mathrm{Co}+\mathrm{C}=0.00677 ; \mathrm{Ao}=40.3 ;$ Gaussiano $\mathrm{Co}=0.01000 ; \mathrm{Co}+\mathrm{C}=17.11000 ; \mathrm{Ao}=20.5$;

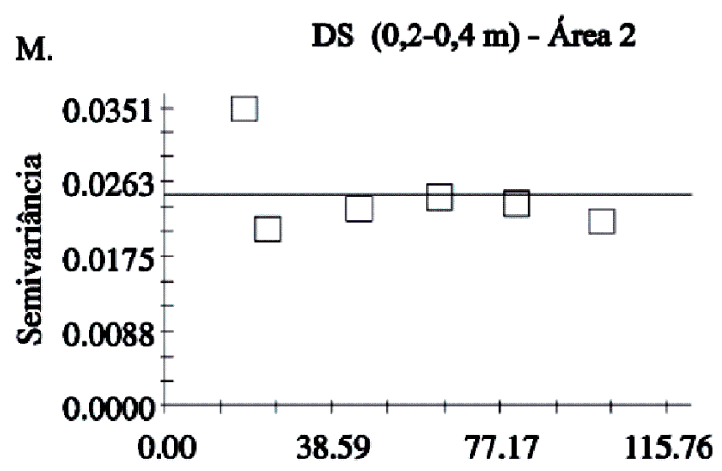

N.

$\theta(0,2-0,4 \mathrm{~m})-$ Área 2

Linear $\mathrm{Co}=0.02495 ; \mathrm{Co}+\mathrm{C}=0.02495 ; \mathrm{Ao}=100.24$;

o.

PT $(0,2-0,4 \mathrm{~m})$ - Área 2

$P$.

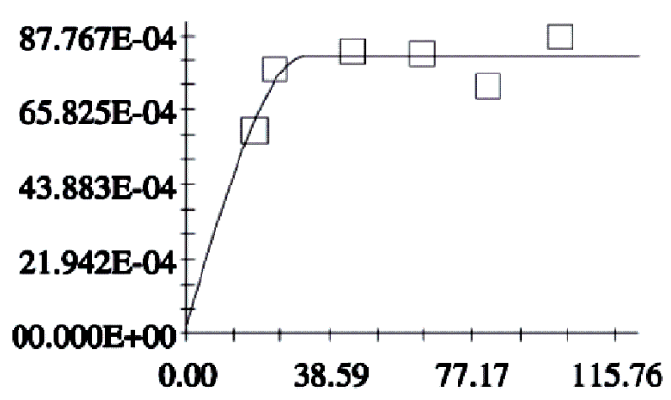

Esférico $\mathrm{Co}=0.00035 ; \mathrm{Co}+\mathrm{C}=0.00820 ; \mathrm{Ao}=32.1$;

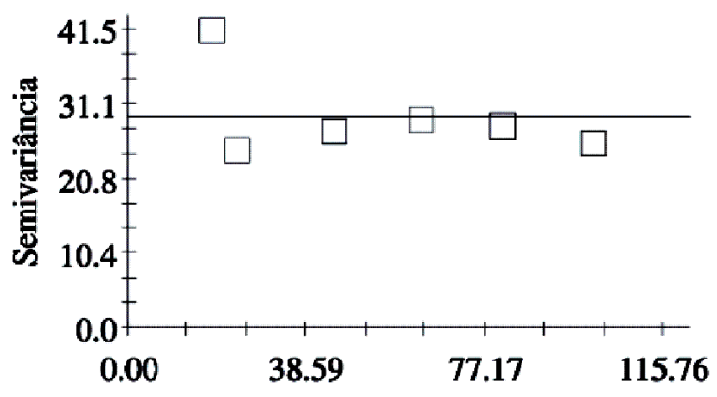

Linear $\mathrm{Co}=29.54947 ; \mathrm{Co}+\mathrm{C}=29.54947 ; \mathrm{Ao}=100.24$; 
Continuação da Figura 1

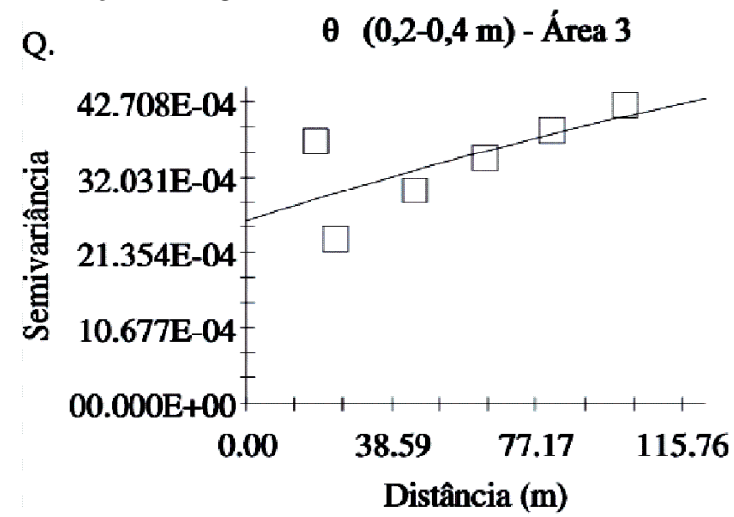

Q.

Distância (m)
R. PT $(0,2-0,4 \mathrm{~m})-$ Área 3

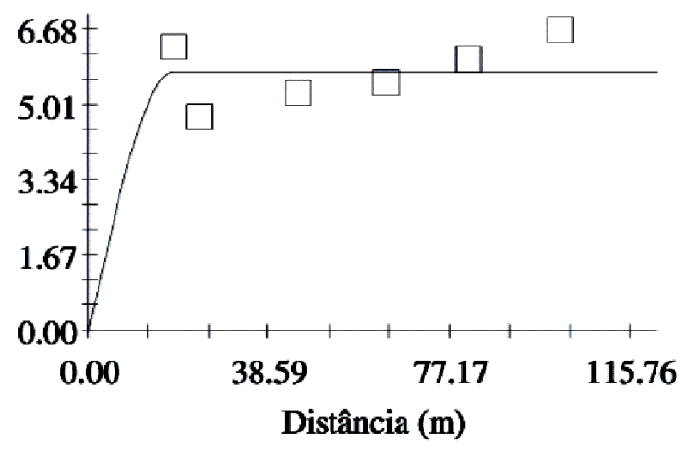

Exponencial $\mathrm{Co}=0.00262 ; \mathrm{Co}+\mathrm{C}=0.00793 ; \mathrm{Ao}=310.0 ; \quad$ Esférico $\mathrm{Co}=0.01000 ; \mathrm{Co}+\mathrm{C}=5.77000 ; \mathrm{Ao}=18.2$;

Figura 1. Semivariogramas para as variáveis DS - densidade do solo $\left(\mathrm{g} \mathrm{cm}^{-3}\right), \theta$ - umidade do solo $\left(\mathrm{m}^{3} \mathrm{~m}^{-3}\right)$ e PT porosidade total (\%) nas profundidades de 0-0,2 m e 0,2-0,4 m das três áreas experimentais

A porosidade total teve maior ajuste no modelo gaussiano (Figura 1L, 1C e 1F) e apresentou ainda modelo esférico (Figura 1R), linear (Figura 1O) e exponencial (Figura 1I), concordando com Cruz et al. (2010), Souza et al. (2004; 2006a), indicando os modelos exponencial e gaussiano como os de maior ocorrência para atributos do solo.

O alcance variou de 10,3 m (Figura 1G) a 297,0 m (Figura 1H) na camada de $0-0,2 \mathrm{~m}$ e de $18,2 \mathrm{~m}$ (Figura $1 \mathrm{R}$ e $1 \mathrm{P}$ ) até $310,0 \mathrm{~m}$ (Figura 1Q) na camada de 0,2-0,4 m.

A fim de analisar o grau da dependência espacial dos atributos físicos em estudo, utilizou-se a classificação de Cambardella et al. (1994), considerada dependência espacial forte em semivariogramas que têm efeito pepita a $25 \%$ do patamar, moderada quando está entre 25 e $75 \%$ e fraca se maior que $75 \%$.

Analisando o grau de dependência espacial dos semivariogramas presentes na Tabela 3, observa-se que, apesar da ocorrência do efeito pepita, as demais variáveis apresentaram, nas duas profundidades, forte dependência espacial, de acordo com a classificação de Cambardella et al. (1994).

Os autores afirmam ainda, que quando as variáveis apresentam forte dependência espacial estarão mais influenciadas por propriedades intrínsecas do solo, como material de origem, relevo, clima, organismos e tempo, ou seja, os chamados fatores de formação.

De acordo com Campos et al. (2008), o alcance indica o limite da dependência espacial da variável, istoé, determinações realizadas a distâncias maiores que o alcance, têm tem distribuição espacial aleatória e, por isso mesmo, são independentes entre si; por outro lado, determinações realizadas em distâncias menores que o alcance são correlacionadas umas às outras, o que permite que se façam interpolações para espaçamentos menores que os amostrados.

Todos os valores de alcance dos atributos dos solos (exclusive os que apresentam efeito pepita) tiveram comportamento regionalizado, sobretudo por apresentarem valores inferiores à distância máxima de amostragem (145,60 $\mathrm{m})$, sendo observado o alcance máximo de 40,30 m da variável umidade na camada de 0,2-0,4 m - Área 1 (Tabela 2), correspondendo a $27,7 \%$ da distância máxima de amostragem.
Os dados ainda apresentaram efeito pepita nulo, indicando que em distâncias de separação pequena entre os pontos, a variabilidade também é pequena, significando que para este parâmetro a distância de estudo foi adequada, concordando com Carvalho et al. (2008).

\section{Conclusões}

1. A palhada encontrada na superfície auxiliou na mantença de bom nível de matéria orgânica, na formação, agregação e estabilidade de poros, impedindo o agrave da densidade;

2. A redução da porosidade total e consecutivo aumento na densidade do solo, principalmente na profundidade de $0,2-0,4$ $\mathrm{m}$, indicam compactação nesta camada, justificada pelo intenso tráfego de veículos junto à ausência de revolvimento do solo;

3. Com os valores apresentados, observa-se necessidade de escarificação nos solos de textura argilo arenosa (1) e areia franca (3).

\section{LITERATURA CITADA}

Berner, P. G M.; Vieira, S. R.; Lima, E.;Anjos, L. H. C. Variabilidade espacial de propriedades físicas e químicas de um Cambissolo sob dois sistemas de manejo de cana-de-açúcar. Revista Brasileira de Ciência do Solo, v.31, p.415-422, 2007.

Braida, J. A.; Reichert, J. M.; Reinert, D. J.; Veiga, M. Teor de carbono orgânico e a susceptibilidade à compactação de um Nitossolo e um Argissolo. Revista Brasileira de Engenharia Agrícola e Ambiental, v.14, p.131-139, 2010.

Cambardella, C. A.; Moorman, T. B.; Novak, J. M.; Parkin, T. B.; Karlen, D. L.; Turco, R. F.; Konopka, A. E. Field-scale variability of soil properties in Central Iowa soils. Soil Science Society America Journal, v.58, p.1501-1511, 1994.

Camilotti, F.; Andrioli, I.; Dias, F. L. F.; Casagrande, A. A.; Silva, A. R.; Mutton, M. A.; Centurion, J. F. Efeito prolongado de sistemas de preparo do solo com e sem cultivo de soqueira de cana crua em algumas propriedades físicas do solo. Engenharia Agrícola, v.25, p.189-198, 2005. 
Campos, M. C. C.; Marques Júnior, J.; Pereira, G. T.; Souza, Z. M.; Barbieri, D. M. Aplicação de adubo e corretivo após o corte da cana-planta utilizando técnicas geoestatísticas. Ciência Rural, v.38, p.974-980, 2008.

Carlesso, R.; Zimmermann, F. L. Água no solo: Parâmetros para o dimensionamento de sistemas de irrigação. Santa Maria: UFSM, 2000. 88p.

Carvalho, L. A.; Mariano Neto, V. J.; Silva, L. F.; Pereira, J. G.; Nunes, W. A. G. A.; Chaves, C. H. C. Resistência mecânica do solo à penetração (RMP) sob cultivo de cana-de-açúcar, no município de Rio Brilhante-MS. Revista Agrarian, v.1, p.7-22, 2008.

Centurion, J. F.; Freddi, O. S.; Aratani, R. G.; Metzner, A. F. M.; Beutler, A. N.; Andrioli, I. Influência do cultivo da cana-deaçúcar e da mineralogia da fração argila nas propriedades físicas de Latossolos Vermelhos. Revista Brasileira de Ciência do Solo, v.31, p. 199-209, 2007.

Corá, J. E.; Beraldo, J. M. Variabilidade espacial de atributos do solo antes e após calagem e fosfatagem em doses variadas na cultura de cana-de-açúcar. Engenharia Agrícola, v.26, p.374-387, 2006.

Costa, F. S.; Bayer, C.; Albuquerque, J. A.; Fontoura, S. M. V. Aumento de matéria orgânica num Latossolo Bruno em plantio direto. Ciência Rural, v.34, p.587-589, 2004.

Cruz, A. C. R.; Pauletto, E. A.; Flores, C. A.; Silva, J. B. Atributos físicos e carbono orgânico de um Argissolo Vermelho sob sistemas de manejo. Revista Brasileira de Ciência do Solo, v.27, p.1105-1112, 2003.

Cruz, J. S.; Assis Júnior, R. N.; Matias, S. S. R.; Tamayo, J. H. C.; Tavares, R. C. Análise espacial de atributos físicos e carbono orgânico em Argissolo Vermelho-Amarelo cultivado com cana-de-açúcar. Ciência e Agrotecnologia, v.34, p.361-368, 2010.

EMBRAPA - Empresa Brasileira de Pesquisa Agropecuária. Manual de métodos de análise de solo. Centro Nacional de Pesquisa de Solo. 2.ed. Rio de Janeiro: Embrapa CNPS, 1997. 212p.

EMBRAPA - Empresa Brasileira de Pesquisa Agropecuária. Sistema brasileiro de classificação de solos. 2.ed. Brasília: Embrapa Produção de Informação. Rio de Janeiro: Embrapa Solos, 2006. 306p.

Fernandes, J.; Ripoli, T. C.; Millan, M. A. A compactação do solo e a brotação das soqueiras. Álcool \& Açúcar, v.3, p.12-17, 1983.

Figueiredo, L. H. A.; Dias Júnior, M. S.; Ferreira, M. M. Umidade crítica de compactação e densidade do solo máxima em resposta a sistemas de manejo num Latossolo Roxo. Revista Brasileira de Ciência do Solo, v.24, p.487-493, 2000.

Gamma Design Software. Geoestatistics for the environmental sciences (version 7.0 for windows). Michigan, Plainwell: 2004. CD-Rom.

Gontijo, I.; Dias Junior, M. S.; Guimarães, P. T. G.; Araújo Júnior, C. F.; Atributos físico-hídricos de um Latossolo de cerrado em diferentes posições de amostragem na lavoura cafeeira. Revista Brasileira de Ciência do Solo, v.32, p.2227-2234, 2008.

Iaia, A. M.; Maia, J. C. S.; Kim, M. E. Uso do penetrômetro eletrônico na avaliação da resistência do solo cultivado com cana-de-açúcar. Revista Brasileira de Engenharia Agrícola e Ambiental, v.10, p.523-530, 2006.
Kiehl, E. J. Manual de edafologia: relação solo-planta. São Paulo: Ceres, 1979. 262p.

Klein, V. A.; Libardi, P. L. Densidade e distribuição do diâmetro dos poros de um Latossolo Vermelho, sob diferentes sistemas de uso e manejo. Revista Brasileira de Ciência do Solo, v.26, p.857-867, 2002.

Klein, V. A.; Libardi, P. L.; Silva, A. P. Resistência mecânica do solo à penetração sob diferentes condições de densidade $\mathrm{e}$ teor de água. Engenharia Agrícola, v. 18, p.45-54, 1998.

Libardi, P. L. Dinâmica da água no solo. São Paulo: USP, 2005. $335 \mathrm{p}$.

Marsili, A.; Servadio, P.; Pagliai, M.; Vignozzi, N. Charges of some physical properties of a clay soil following passage of ruber-and metal-tracked tractors. Soil \& Tillage Research, v.49, p.185-199, 1998.

Mello, G.; Bueno, C. R. P.; Pereira, G. T. Variabilidade espacial das propriedades físicas e químicas do solo em áreas intensamente cultivadas. Revista Brasileira de Engenharia Agrícola eAmbiental, v.10, p.294-305, 2006.

Michelon, C. J. Qualidade física dos solos irrigados do Rio Grande do Sul e do Brasil Central. Santa Maria: UFSM, 2005. 92p. Dissertação Mestrado

Ortiz, J. L. Emprego do geoprocessamento no estudo da relação entre potencial produtivo de um povoamento de eucalipto e atributos do solo e do relevo. Piracicaba: ESALQ, 2003. 205p. Dissertação Mestrado

Pacheco, E. P. Estudo da compressibilidade e qualidade de um Argissolo Amarelo cultivado com cana-de-açúcar nos tabuleiros costeiros de Alagoas. Recife: UFRP, 2010. 118p. Tese Doutorado

Paulino, A. F.; Medina, C. C.; Azevedo, M. C. B.; Silveira, K. R. P; Trevisan, A. A.; Murata, I. M. Escarificação de um Latossolo Vermelho na pós-colheita de soqueira de cana-de-açúcar. Revista Brasileira de Ciência doSolo, v.28, p.911-917, 2004.

Ramirez-López, L.; Sánchez, A. R.; Tamayo, J. H. C. Variabilidad espacial de atributos físicos de un typic haplustox de los llanos orientales de Colômbia. Engenharia Agrícola, v.28, p.55-63, 2008.

Reichardt, K.; Timm, L. C. Solo, planta e atmosfera: conceito, processo e aplicações. Barueri: Manole, 2004, 478p.

Reichert, J. M.; Suzuki, L. E. A. S.; Reinert, D. J. Compactação do solo em sistemas agropecuários e florestais: identificação, efeitos, limites críticos e mitigação. Tópicos Ciência Solo, v.5 p.49-134, 2007.

Sanchez, R. B.; Marques Júnior, J.; Souza, Z. M.; Pereira, G. T.; Martins Filho, M. V. Variabilidade espacial de atributos do solo e de fatores de erosão em diferentes pedoformas. Bragantia, v.68, p.1095-1103, 2009.

Statsoft, Statistica for Windows (data analysis software system), version 4.3 Statsoft, Oklahoma, 2005.

Shapiro, S. S.; Wilk, M. B. An analysis of variance test for normality (complete samples). Biometrika, v.52, p.591-611, 1965.

Silva, A. J. N.; Cabeda, M. S. V. Compactação e compressibilidade do solo sob sistemas de manejo e níveis de umidade. Revista Brasileira de Ciência do Solo, v.30, p.921-930, 2006. 
Silva, A. J. N.; Cabeda, M. S. V.; Carvalho, F. G. Matéria orgânica e propriedades físicas de um Argissolo Amarelo Coeso sob sistemas de manejo com cana-de-açúcar. Revista Brasileira de Engenharia Agrícola e Ambiental, v.10, p.579-585, 2006.

Silva, A. J. N.; Ribeiro, M. R. Caracterização de um Latossolo Vermelho-Amarelo sob cultivo contínuo de cana-de-açúcar no estado de Alagoas: atributos morfológicos e físicos. Revista Brasileira de Ciência do Solo, v.21, p.677-684, 1997.

Silva, V. R.; Reinert, D. J.; Reichert, J. M. Resistência mecânica do solo à penetração influenciada pelo tráfego de uma colhedora em dois sistemas de manejo do solo. Ciência Rural, v.30, p.795-801, 2000.

Siqueira, G. M.; Vieira, S. R.; Ceddia, M. B. Variabilidade de atributos físicos do solo determinados por métodos diversos. Bragantia, v.67, p.203-211, 2008.

Souza, Z. M.; Campos, M. C. C.; Cavalcante, Í. H. L.; Marques Júnior, J.; Cesarin, L. G.; Souza, S. R. Dependência espacial da resistência do solo à penetração e do teor de água do solo sob cultivo contínuo de cana-de-açúcar. Ciência Rural, v.36, p.128-134, 2006 a.

Souza, Z. M.; Marques Júnior, J.; Pereira, G. T. Variabilidade espacial de atributos físicos do solo em diferentes formas do relevo sob cultivo de cana-de-açúcar. Revista Brasileira de Ciência do Solo, v.28, p.953-964, 2004.

Souza, Z. M.; Marques Júnior, J.; Pereira, G. T. Geoestatística e atributos do solo em áreas cultivadas com cana-de-açúcar. Ciência Rural, v.40, p.69-77, 2010.

Souza, Z. M.; Marques Júnior, J.; Pereira, G. T.; Montanari, R.; Campos, M. C. C. Amostragem de solo para determinação de atributos químicos e físicos em área com variação nas formas do relevo. Revista Científica, v.34, p.249-256, $2006 b$.
Souza, Z. M.; Prado, R. M.; Paixão, A. C. S.; Cesarin, L. G. Sistemas de colheita e manejo da palhada de cana-de-açúcar. Pesquisa Agropecuária Brasileira, v.40, p.271-278, 2005.

Spadoto, A. F. Desenvolvimento de programa computacional aplicado ao empacotamento do palhiço de cana-de-açúcar. Botucatu: UNESP, 2008. 62p. Dissertação Mestrado

Stone, L. F.; Silveira, P. M. Efeitos do sistema de preparo e da rotação de culturas na porosidade e densidade do solo. Revista Brasileira de Ciência do Solo, v.25, p.395-401, 2001.

Timm, L. C.; Reichardt, K.; Oliveira, J. C. M.; Cassaro, F. A. M.; Tominaga, T. T.; Bacchi, O. O. S.; Dourado Neto, D.; Nielsen, D. R. State-space approach to evaluate the relation between soil physical and chemical properties. Revista Brasileira de Ciência do Solo, v.28, p.49-58, 2004.

Tormena, C. A.; Barbosa, M. C.; Costa, A. C. S. Densidade, porosidade e resistência a penetração em Latossolo cultivado sob diferentes sistemas de preparo do solo. Scientia Agrícola, v.59, p.795-801, 2002.

Tormena, C. A.; Roloff, G.; Sá, J. C. M. Propriedades físicas do solo sob plantio direto influenciado por calagem, preparo inicial e tráfego. Revista Brasileira de Ciência do Solo, v.22, p.301-309, 1998.

van Raij, B.; Andrade, J. C.; Cantarella H.; Quaggio, J. A. Análise química para avaliação da fertilidade de solo tropicais. Campinas: Instituto Agronômico, 2001. 285p.

Vieira, M. L.; Klein, V. A. Propriedades físico-hídricas de um Latossolo Vermelho submetido a diferentes sistemas de manejo. Revista Brasileira de Ciência do Solo, v.31, p.16371646, 2007.

Warrick, A. W.; Nielsen, D. R. Spatial variability of physical properties in the field. In: Hillel, D. (ed.). Applications of soil physics. New York: Academic Press, 1980. p.319-344. 